\title{
Comparison of MPPT strategies for solar modules
}

\author{
M. Calavia ${ }^{1}$ J.M. Perié ${ }^{1}$ J.F. Sanz ${ }^{2}$ and J. Sallán ${ }^{2}$ \\ ${ }^{1}$ CIRCE Institute. C/María de Luna 3. 50018 Zaragoza. \\ Phone number: +34 976761000, ext: 5164. Fax number: +34 976 976732078. e-mail: marta.calavia@unizar.es; \\ jmperie@unizar.es
}

${ }^{2}$ CIRCE Institute - Universidad de Zaragoza. Electrical Engineering Department. C/María de Luna 3. 50018 Zaragoza. Phone number: +34 976762403/2399. Fax number: +34 976 976762226. e-mail: jfsanz@unizar.es; jsallan@unizar.es

\begin{abstract}
The paper studies the behavior of three different Maximum Power Point Tracking (MPPT) strategies applied to solar modules: three-point perturb and observe, fixed-step incremental conductance, and variable-step incremental conductance.

It starts with a brief description of each method and then it compares them considering three criteria: simplicity of implementation, capability to follow irradiance variations and sensitivity to noise in the required measurements. Diverse simulations have been performed and the main results are presented.
\end{abstract}

\section{Key words}

Solar energy, maximum power point tracking, efficiency comparison, measurement noise.

\section{Introduction}

MPPT algorithms are used in solar modules in order to make the system operate with maximum efficiency. Their main mission is to adapt the operation point of the module, usually modifying its output voltage, in order to adapt the system to different irradiance conditions, and also to temperature variations.

Different MPPT techniques can be found in the literature: perturb and observe [1], [4], [5], fixed-step incremental conductance [4], [5], and variable-step incremental conductance [2], [3]. The present paper compares the results obtained applying these three methods to a $9600 \mathrm{~W}$ array composed of $48200 \mathrm{~W}$ modules (8 in series, 6 in parallel), with the characteristics shown in table I.

All the implemented algorithms work independently of the parameters and characteristic curves of the PV modules. Thus, simple systems are achieved, since the temperature and irradiance sensors have not to be installed in the panels. In addition, the systems are more robust, because the optimum operation point is reached although parametric drift appears on the components.
Table I. - Solar module characteristics.

\begin{tabular}{|l|c|}
\hline Rated Power $\left(\mathrm{P}_{\max }\right)$ & $200 \mathrm{~W}$ \\
\hline Maximum Power Voltage & $55.8 \mathrm{~V}$ \\
\hline Maximum Power Current & $3.59 \mathrm{~A}$ \\
\hline Open Circuit Voltage $\left(\mathrm{V}_{\mathrm{OC}}\right)$ & $68.7 \mathrm{~V}$ \\
\hline Short Circuit Current $\left(\mathrm{I}_{\mathrm{SC}}\right)$ & $3.83 \mathrm{~A}$ \\
\hline Temperature coefficient $\left(\mathrm{P}_{\max }\right)$ & $-0.29 \% /{ }^{\circ} \mathrm{C}$ \\
\hline Temperature coefficient $\left(\mathrm{V}_{\mathrm{OC}}\right)$ & $-0.172 \mathrm{~V} /{ }^{\circ} \mathrm{C}$ \\
\hline Temperature coefficient $\left(\mathrm{I}_{\mathrm{SC}}\right)$ & $0,88 \mathrm{~mA} /{ }^{\circ} \mathrm{C}$ \\
\hline
\end{tabular}

\section{System design}

The studied photovoltaic system is integrated with several generation and storage elements of renewable energies. This integration is made up of a common DClink with all the subsystems connected to it. The reason for this configuration is double: First, the interconnected systems complexity is lower, because the DC-link voltage and current are the only electrical magnitudes to be controlled, instead of phase or frequency. Second, the existing storage systems work with direct current, therefore the storage in capacitors, batteries and ultracapacitors becomes easier.

However, since diverse storage elements with different states of charge exist in the DC-link, a constant voltage value cannot be preset to the DC-link. In fact, this voltage is used as a signal that represents the power balance in the system, indicating when the storage elements have to supply or absorb energy. In this manner, a scalable system conformed of heterogeneous systems is achieved with mutual control independence.

Therefore, unlike other photovoltaic systems that use batteries as the only storage system, and in which the voltage varies slightly only due to the state of charge; the present system is going to have bigger variations of the DC-link voltage that the solar converter will not be able to control. 


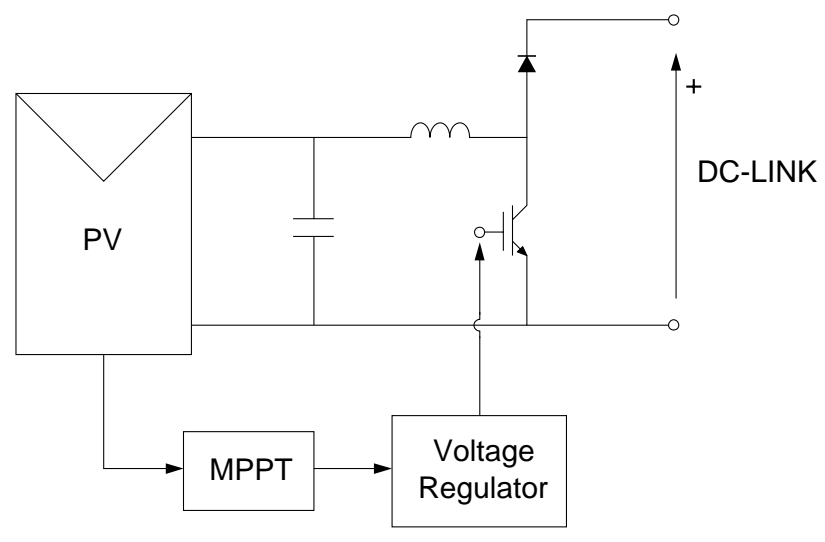

Fig. 1. System structure.

In this case, a voltage regulator is needed to make the MPPT insensitive to DC-link voltage changes. Unlike the existing algorithms found in the literature, in which the MPPT acts directly on the duty cycle of the converter, in the present system it modifies the desired voltage of the capacitor located in parallel with the photovoltaic panels. Then, the controller varies the duty cycle taking into account the DC-link voltage to reach the value desired by the MPPT. Therefore, if a variation suddenly occurs in the DC-link voltage, the controller will compensate it, and neither the algorithm nor the panels will notice the change.

The implemented voltage regulator is a discrete PID with a response time of $5 \mathrm{~ms}$ that is enough to grant the controllability of the system with a $4 \mathrm{kHz}$ switching frequency. It also prevents the saturation of the controller when the MPPT changes sharply the desired voltage.

On the other hand, since the input of the boost converter is connected to one of the terminal of the inductor, and a parallel capacitor is required to maintain the voltage level, a series LC circuit without damping appears. To avoid resonance two basic techniques exist in the literature: passive damping and active damping.

Passive damping, the simplest one, consists in a physical resistor that is connected, for example, in series with the inductor or the capacitor. The purpose is to increase the damping factor, and then, avoid the resonance effect of the second order system. The problem of this method are the losses due to the resistor, a drawback that is undesirable for a system trying to maximize the obtained energy.

The active damping takes advantage of the output voltage modulation at the IGBT collector to dynamically compensate the oscillating behaviour of the LC filter. Although many active damping techniques exist in the literature, the virtual resistor [5], [6], has been chosen. The reason is the simplicity of implementation and the robustness to parametrical drift of the LC filter elements. This method consists in modifying the average output voltage of the transistor in the same way as the voltage would drop due to a virtual resistor in series with the inductor. Since there is an inductor current sensor, this measure only has to be multiplied by the virtual resistor value, and the result has to be added to the output voltage level from the PID controller.

\section{Strategy description}

This section describes three MPPT methods: three-point perturb and observe, fixed-step incremental conductance, and variable-step incremental conductance.

\section{A. Three-point perturb and observe}

This algorithm consists in varying the voltage of the capacitor located in parallel with the solar modules, measuring the power obtained in each situation. The algorithm takes three different voltages $\left(\mathrm{V}_{\mathrm{a}}, \mathrm{V}_{\mathrm{b}}, \mathrm{V}_{\mathrm{c}}\right)$ and, as a result, it obtains three generated power values $\left(\mathrm{P}_{\mathrm{a}}\right.$, $\left.\mathrm{P}_{\mathrm{b}}, \mathrm{P}_{\mathrm{c}}\right)$.

Depending on the results obtained, it can be determined if the operation points are located to the right or to the left of the maximum power point, and the module voltage must be increased or decreased, respectively (Fig. 2). If the highest generated power value corresponds to $V_{a}$, an optimum situation has been found.

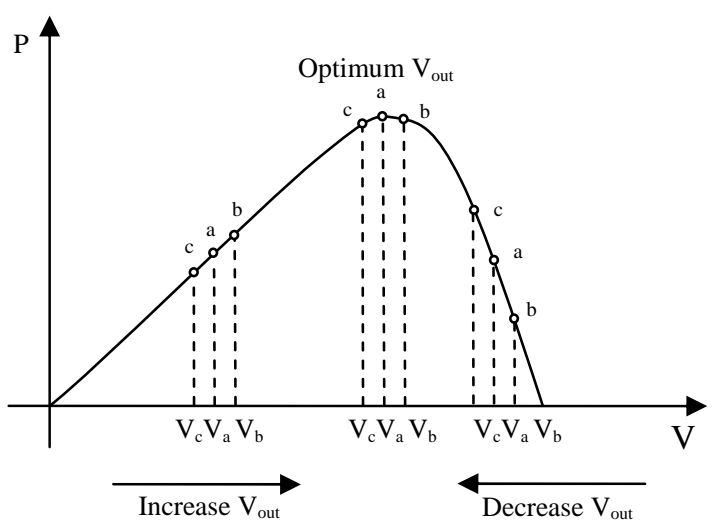

Fig. 2. Operation of the 3-point perturb and observe algorithm.

Once the optimum point is reached, there is still some ripple in the generated power, since the algorithm keeps modifying the output voltage to track possible deviations due, for instance, to irradiance variations.

With this algorithm there is a compromise between the maximum power point tracking capabilities, and the voltage ripple due to the perturbations when the most efficient point is reached. If a too high value is taken, the steady state steps will introduce an excessive ripple on the power supplied by the panels, which has negative repercussions on the efficiency. On the other hand, if the step is too small, a slow response to irradiance changes is obtained, which will also lead to an efficiency reduction, especially in cloudy and windy days.

For simulations, a 2 volt step has been chosen, which is applied every $20 \mathrm{~ms}$. This is enough time for the regulator to reach the steady state voltage. Taking into account that it is a three step algorithm, this means that it 
has a reference voltage $\left(\mathrm{V}_{\mathrm{a}}\right)$ slew-rate of 2 volt every 60 ms, about 33 volt per second.

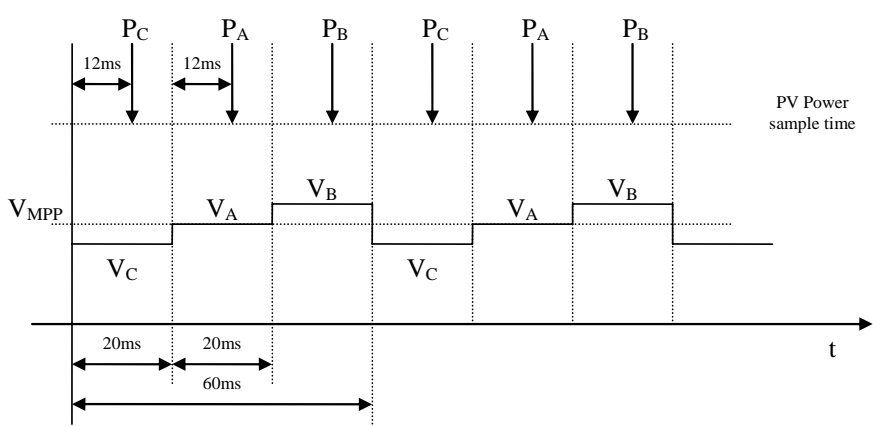

Fig. 3. Temporal evolution of the $\mathrm{P} \& \mathrm{O}$ algorithm.

\section{B. Fixed-step incremental conductance}

The incremental conductance algorithm consists in studying the slope of the module power-voltage curve.

The control criteria that increase, decrease or maintain the MPPT voltage is based on the next equation:

$$
\frac{d P}{d V}=\frac{d(V \cdot I)}{d V}=I \frac{d V}{d V}+V \frac{d I}{d V}=I+V \frac{d I}{d V}
$$

The power will be the highest when the power-voltage slope is null. Using the previous expression, the next conditions are obtained:

$$
\begin{aligned}
\text { - } \frac{d I}{d V}>-\frac{I}{V} \Rightarrow \frac{d P}{d V}>0 \\
\text { - } \frac{d I}{d V}<-\frac{I}{V} \Rightarrow \frac{d P}{d V}<0
\end{aligned}
$$

If the slope is positive, the maximum is situated to the right, and the voltage must be increased. On the contrary, if the slope is negative, the optimum is located to the left, and the voltage must be decreased (Fig. 4).

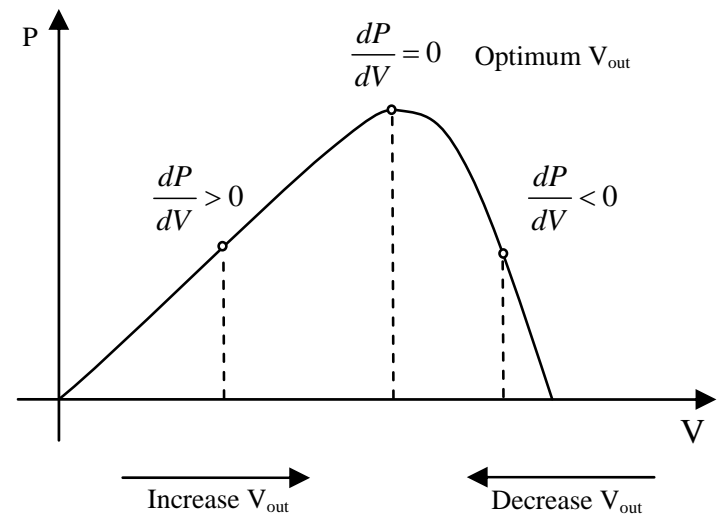

Fig. 4. Operation of the fixed-step incremental conductance algorithm.
The power derivative is calculated from the voltage and current instantaneous values. The differences of these variables are obtained using the intrinsic ripple of the converter. Since the present system is conformed by a $\mathrm{DC} / \mathrm{DC}$ boost converter, the capacitor voltage ripple has a fundamental frequency equal to the IGBT switching frequency, $4 \mathrm{kHz}$. This is why the capacitor in parallel with the panels is especially sized for obtaining enough signal/noise sensitivity in the measurement of the differences. Another significant parameter for getting a good signal/noise ratio is the sample time. In Fig. 5 the characteristic voltage and current ripple are shown.

Taking into account that the voltage and current measurement have to be simultaneous, and that the duty cycle is variable, it is not an easy task to choose a sample time that optimizes the magnitude of both differences, maximizing the signal/noise ratio of the measurement. The chosen sample times have been taken at $(K-0.25) T$ and $K T$, being $T$ the switching period.
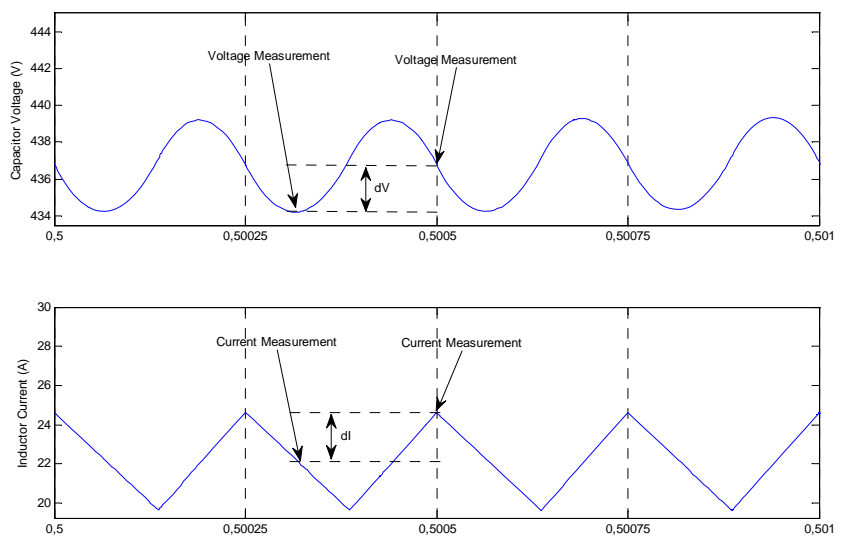

Fig. 5. Voltage and current ripple.

The most important advantage of this method is that, once the optimum point is reached, the voltage remains unchanged, so the steady-state performance is increased.

The incremental conductance MPPT output voltage is evaluated every 250 microseconds; it results in a nearly continuous desired voltage at the regulator input. This fact allows a good tracking of the signal by the controller, providing a robust system for changes of irradiance and temperature.

As with the previous algorithm, the MPPT step must be adjusted to get a good convergence time without losing too much tracking fidelity. The selected step has been $200 \mathrm{mV}$, which is updated every 250 microseconds. With these values, 800 volts per second slew rate is reached. 


\section{Variable-step incremental conductance}

This method is a modification of the one previously described. The difference is the continuous modification of the step, taking into account, not only the sign of the slope of the power-voltage curve, but also its value. It can be noticed that the slope decreases as the operation point gets closer to the optimum point, so the step can be made proportional to this value (Fig. 6).

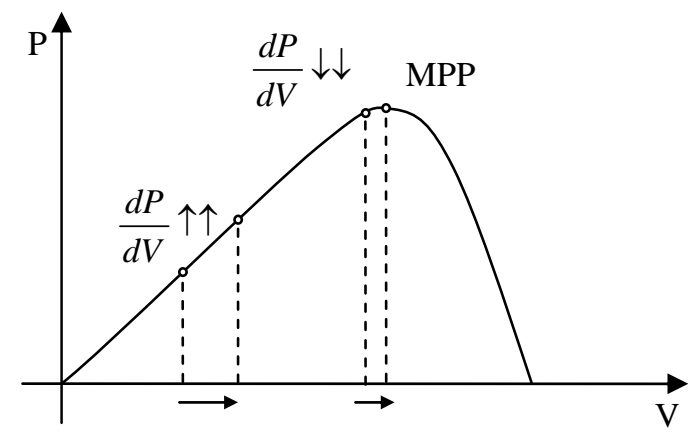

Fig. 6. Operation of the variable-step Inc. Cond. algorithm.

Many methods for defining the gain between the powervoltage slope and the step appear in the literature [4], although it is not easy to define a gain for all the irradiance and temperature conditions. In addition, this algorithm is susceptible to an oscillating behaviour from side to side of the optimum point, if there is a high slope in both points. Likewise, if a transient measurement failure occurs, the obtained difference will be abnormally large, and therefore a spike will appear on the estimated power-voltage slope signal. As a result, the systems will go far away from the optimum operation point. Because of this reason, the algorithm maximum step has been bounded to $10 \mathrm{~V}$. This implies that a $400 \mathrm{kV}$ per second slew-rate can be achieved. The rest of the parameters are adjusted in the same way as in the fixed step algorithm.

\section{Strategy comparison}

This section compares the three studied MPPT algorithms, focusing on two scenarios. In the first one, irradiance varies but there is not any noise in the measurements, with the objective of analyzing the dynamic performance of the three algorithms on irradiance changes. The irradiance function chosen for this scenario is a variable signal between 400 and $1000 \mathrm{~W} / \mathrm{m}^{2}$ with several irradiance changes along the time. In the second one, irradiance is a constant value of $800 \mathrm{~W} / \mathrm{m}^{2}$, but there is some noise in all the system measurements.

In others papers, the algorithms behavior is analyzed without referring to the possible noise effects. However, when these algorithms are implemented in real systems, the measurements are altered by the noise, so that, the results might not be the expected ones.
The type of noise introduced in the simulated system has been white gaussian noise discretely generated with 1 Msps, and with a variance of $10 \mathrm{~mA}$ on the current measures and $100 \mathrm{mV}$ on the voltage ones. These noise levels are usually encountered at environments with power electronics when inexpensive sensors and conditioning circuits are used.

The responses of the algorithms to a sudden irradiation change, when the noise is not present at the measurements, are shown in Fig. 7. It can be noticed that, the Perturb and Observe algorithm has a slower behaviour than the others, due to the lower slew-rate of this method. It can also be perceived the difference between algorithms of incremental conductance. The fixed step method increases the capacitor voltage in a constant and continuous manner; meanwhile, the variable step method converges to the most efficient point with a step that is proportional to the power slope.

Both algorithms respond quickly to the sudden irradiance variation, the variable step method is capable of reaching the optimum point before the fixed step method.

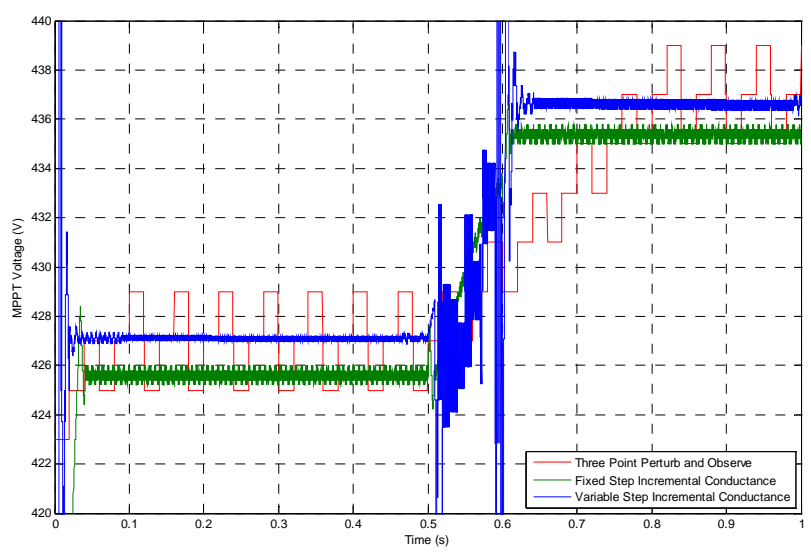

Fig. 7. MPPT voltage with an irradiance variation.

Then, in figures from Fig. 8 to Fig. 10 the MPPT voltages of different algorithms implemented are shown and, also, the extracted power of the solar modules, as well as, their steady-state response when the simulation is performed with and without noise in the measurement.

It can be noticed in Fig. 8 that, when the noise does not exist in the measurements, the Perturb and Observe algorithm modifies the voltage around the most efficient point. However, when the noise is considered in the simulation, an important deviation from the expected behaviour appears. In the same way, the power ripple due to the MPPT voltage fluctuation is shown. This ripple and the caused deviation due to the measurement noise make the system performance worse when this algorithm is used.

The MPPT voltage of the fixed step Incremental Conductance algorithm reaches quickly the steady state and it remains stable in this point as it can be seen in the Fig. 9. However, when the simulation is performed 
introducing noise in the measurements, a small deviation is obtained, which fluctuates around the optimum point due to the wrong differential samples caused by the introduced noise. This deviation is smaller than in the case of the Perturb and Observe algorithm.

It can also be perceived the effect of noise in the obtained power of the solar modules. In the case of the simulation without noise, the reached power is practically constant, although, when the simulation is performed with noise, a ripple of a few watts appears.

Fig. 10 shows how this algorithm is quite affected by noise. Initially, when the simulation is executed without noise, the obtained MPPT voltage is practically constant as in the fixed step method. However, when the system is again simulated with noise, instability appears because the power slope is calculated incorrectly due to noise.
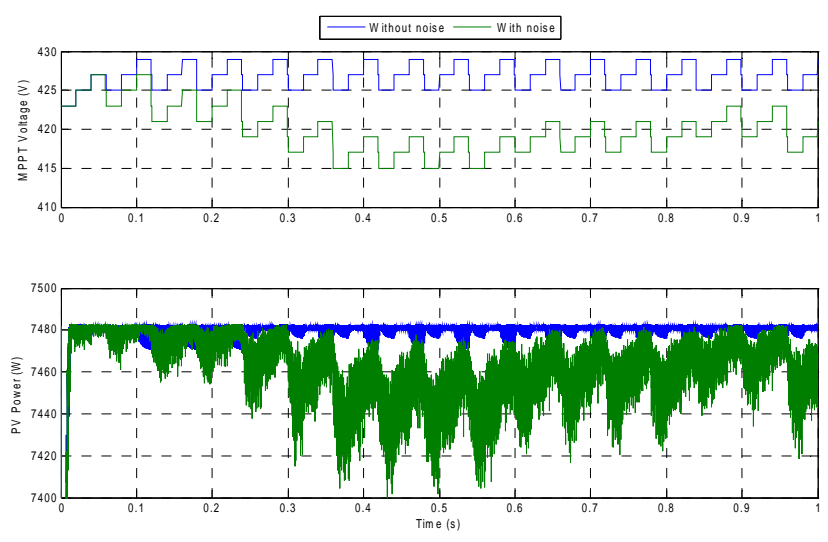

Fig. 8. Three point Perturb and Observe.
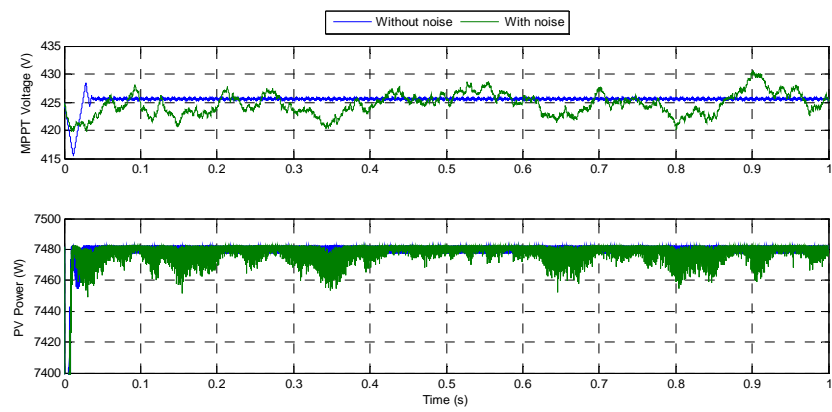

Fig. 9. Fixed Step Incremental Conductance.
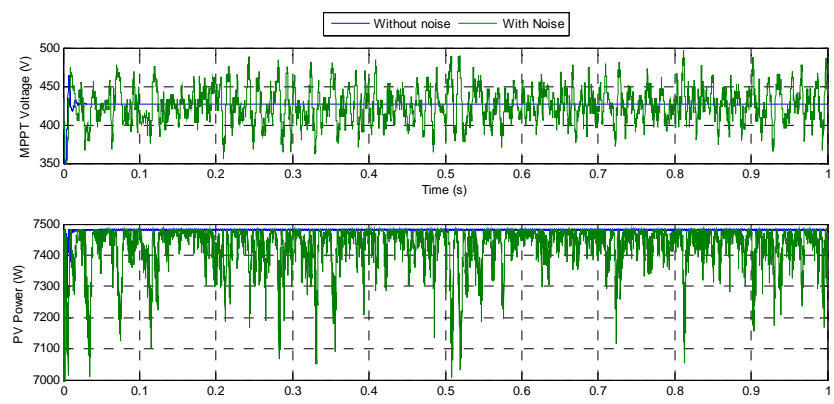

Fig. 10. Variable Step Incremental Conductance.
Fig. 11 shows that the incremental conductance algorithms are the ones extracting more power when an irradiance change occurs. This behavior is due to the better dynamic response of those systems, since they do not have to wait for the output of the controller to reach the steady state value. It can also be noticed that there is almost no difference between the incremental conductance algorithms, although the one using variable step is slightly better.

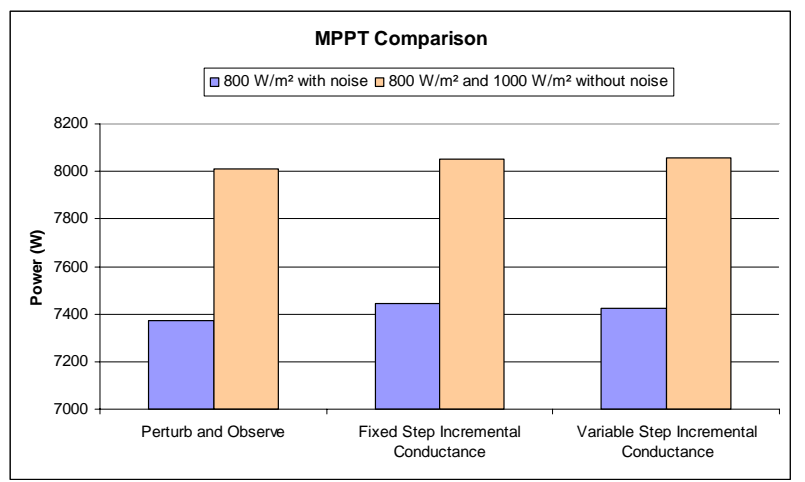

Fig. 11. Generated power comparison for the three MPPT algorithms in the two analysed scenarios.

However, when the algorithms have to operate with noisy signals, the fixed-step incremental conductance algorithm gives a better result, due to its higher robustness, since it only needs the sign of the derivative of the generated power.

Finally, it can also be noticed that the perturb and observe algorithm is the worst in both cases, showing as only advantage the simplicity of its implementation.

\section{Conclusion}

The paper studies three MPPT methods and analyses their behavior when they have to follow a sudden change in irradiance or when significant noise is present in the voltage and current measurements.

The first method, three-point perturb and observe, is the simplest to implement, but it shows the worst behavior in power tracking both with and without noise.

The two incremental conductance methods are more difficult to implement, but they have better power tracking characteristics (slightly better for the variablestep system). The fixed-step method is the one showing higher immunity to noise measurements, since it only requires the evaluation of the sign of the generated power derivative.

Therefore, in the real systems, the existent noise in the measurements, although this is very low, has a significant effect in the performance of the algorithms compared with obtained results in ideal simulations, reducing the global system performance. 


\section{References}

[1] J.A. Jiang, T.L. Huang, Y.T. Hsiao and C.H. Chen, "Maximum Power Tracking for Photovoltaic Power Systems", Tamkang Journal of Science and Engineering, Vol. 8, No 2, 2005, pp. 147-153.

[2] K.H. Hussein, 1. Muta, 1. Hoshino and M. Osakada, "Maximum photovoltaic power tracking: an algorithm for rapidly changing atmospheric conditions", IEE Proc. Gener. Trans. and Distrib., Vol. 142, No. 1 , 1995, pp. 59-64.

[3] F. Luo , P. Xu , Y. Kang and S. Duan, “A Variable Step Maximum Power Point Tracking Method Using Differential Equation Solution”, Second IEEE Conference on Industrial Electronics and Applications, 2007, pp. 2259-2263.
[4] F.Liu, S. Duan, F. Liu, B. Liu, and Y. Kang, "A Variable Step Size INC MPPT Method for PV Systems", IEEE Transactions on Industrial Electronics, Vol. 55, No. 7, July 2008, pp. 2622-2628.

[5] P. A. Dahono, "A Control Method to Damp Oscillation in the Input LC Filter of AC-DC PWM Converters", IEEE Power Electronics Specialists Conference, 2002, vol. 4, pp. 1630-1635.

[6] P.A. Dahono, Y.R. Bahar, Y. Sato, T. Kataoka, "Damping of transiente oscillations on the output LC filter of PWM inverters by using a virtual resistor”, IEEE International Conference on Power Electronics and Drive Systems, 2001, Volume 1, 22-25 Oct. 2001 pp. 403 407. 